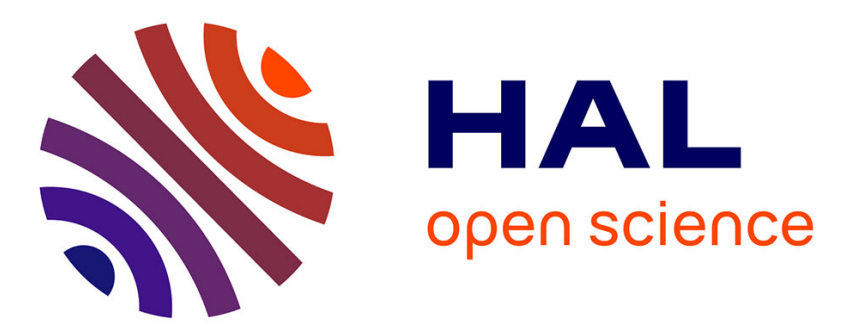

\title{
Identification of TRIO-GEFD1 chemical inhibitors using the yeast exchange assay.
}

Anne Blangy, Nathalie Bouquier, Cécile Gauthier-Rouvière, Susanne Schmidt, Anne Debant, Jean-Paul Leonetti, Philippe Fort

\section{> To cite this version:}

Anne Blangy, Nathalie Bouquier, Cécile Gauthier-Rouvière, Susanne Schmidt, Anne Debant, et al.. Identification of TRIO-GEFD1 chemical inhibitors using the yeast exchange assay.. Biology of the Cell, 2006, 98 (9), pp.511-22. 10.1042/BC20060023 . hal-00189369

\section{HAL Id: hal-00189369 https://hal.science/hal-00189369}

Submitted on 20 Nov 2007

HAL is a multi-disciplinary open access archive for the deposit and dissemination of scientific research documents, whether they are published or not. The documents may come from teaching and research institutions in France or abroad, or from public or private research centers.
L'archive ouverte pluridisciplinaire HAL, est destinée au dépôt et à la diffusion de documents scientifiques de niveau recherche, publiés ou non, émanant des établissements d'enseignement et de recherche français ou étrangers, des laboratoires publics ou privés. 
Identification of TRIO-GEFD1 chemical inhibitors using the Yeast Exchange Assay.

${ }^{*}$ Anne Blangy, *Nathalie Bouquier, ${ }^{*}$ Cécile Gauthier-Rouvière, *Susanne Schmidt, *Anne Debant, "Jean-Paul Leonetti and *Philippe Fort.

${ }^{*}$ Centre de Recherches en Biochimie Macromoléculaire, CNRS FRE2593, 1919 route de Mende, 34293 Montpellier Cedex 5 and ${ }^{\#}$ Faculté de Pharmacie, CPBS CNRS UMR 5160, 15 avenue Charles Flahault, B.P. 14491, 34093 MONTPELLIER Cedex 5 .

${ }^{\S}$ Corresponding author:

Anne Blangy

Centre de Recherches en Biochimie Macromoléculaire, CNRS FRE 2593 1919 Route de Mende. 34293 Montpellier Cedex 5. France

Tel: 33467613422

Fax: 33467521559

e-mail: anne.blangy@crbm.cnrs.fr

Running title: How to identify RhoGEF inhibitors.

Key words: RhoG, exchange factor, yeast, screen, nucleotide. 


\section{Abbreviations}

AMCA: amino-methyl coumarin

CPEPD: 1,1'-(2-chloro-1,4-phenylene)bis(1H-pyrrole-2,5-dione)

GEF: Guanine nucleotide exchange factor

Mant: N-methylanthraniloyl

NPPD: 1-(3-nitrophenyl)-1H-pyrrole-2,5-dione

PEPD: 1,1'-(1,2-phenylene)bis(1H-pyrrole-2,5-dione)

RhoGEF: Rho GTPase guanine nucleotide exchange factor 


\section{Acknowledgements}

We thank Matthias Peter, Jacques Camonis and Philippe Pasero for the gift of reagents and technical advice. We thank Bernard Guibert and Jean-Christophe Zeeh for the preparation of recombinant Arf1 and ARNO. We thank Fanny Malhaire, Audrey Salomon and Pauline Labrousse for technical support. This work was supported by the Centre National de la Recherche Scientifique and by a grant from the Ligue Nationale Contre le Cancer (Equipe labellisée). The authors declare competing financial interests. Yeast strains and screening procedures have been deposited for patent under the accession W005064007A2. 


\section{Abstract \\ Background information}

RhoGTPases are involved in many biological processes and participate in cancer development. Their activation is catalyzed by exchange factors (RhoGEFs) of the Dbl family. RhoGEFs display proto-oncogenic features, thus appearing as candidate targets for anticancer drugs. Dominant negative RhoGTPase mutants have been widely used to block RhoGEF signaling. However, these tools suffer from limitations, due to the high number of RhoGEFs and the complex mechanisms that control RhoGTPase activation.

\section{Results}

RhoG-T17N is a poor inhibitor of its exchange factor TRIO-GEFD1 in vivo: although it binds to TRIO-GEFD1, RhoG-T17N does not block the downstream signaling. Using the Yeast Exchange Assay we show that in the presence of TRIO-GEFD1, RhoGT17N can bind to its effectors, which illustrates how negative mutants may produce misleading interpretations and emphasizes the need of new types of RhoGEF inhibitors. In that prospect, we adapted the Yeast Exchange Assay method to identify RhoGEF inhibitors. Using this novel approach, we screened a 3,500 chemical compound library and identified a potential inhibitor of TRIO-GEFD1. This molecule inhibited TRIO-GEFD1 in vitro. Among the chemical analogs of this compound, we identified two molecules with better inhibitory activity. The three TRIO-GEFD1 inhibitors had no effect on ARHGEF17 and ARNO, two exchange factors for RhoA and Arf1 respectively.

\section{Conclusions}

The development of RhoGEF inhibitors appears as a necessary tool for the study of Rho GTPase signaling pathways. The Yeast Exchange Assay adaptation we present 
here is suitable to screen for chemical or peptide libraries and identify candidate inhibitors. 


\section{1- Introduction.}

Small GTPases of the Rho family regulate actin cytoskeleton dynamics, cell morphology, adhesion, migration, intracellular trafficking, embryonic development, apoptosis and transformation (Etienne-Manneville and Hall, 2002). They are inactive when bound to GDP and active when bound to GTP. When active, they can bind to their effectors and activate downstream signaling cascades. Rho GTPase activation is catalyzed by Guanine Nucleotide Exchange Factors (GEFs) of the Dbl and the CZH/DOCK families. In mammals, there are 65 Dbl-related (Rossman et al., 2005) and 11 DOCK-related proteins (Meller et al., 2005) and each GEF activates one or several GTPases. The exchange domain is responsible for GTPase activation. RhoGEFs contain additional domains involved in lipid and protein binding that are supposed to regulate their localization and biological activity.

The interaction between the GTPase and the exchange factor is a complex mechanism involving multiple domains of either partner. Even though several threedimensional structures of GTPase-GEF complexes have been solved, the exact mechanisms of substrate selectivity by the exchange factors are not known (Erickson and Cerione, 2004; Rossman et al., 2005). Mutant analyses revealed that point mutations at identical positions could have distinct effects depending on the GTPase and the exchange factor involved. For instance, the T37A mutant of RhoA and the equivalent T35A mutant of Cdc42 do not respond to activation by their respective exchange factors Lbc and Cdc24 (Li et al., 1997). In contrast, the T35A mutant of Rac1 still responds to TRIO-GEFD1 (Gao et al., 2001). Similarly, while the F39E mutant of RhoA cannot be activated by Lbc, it retains the ability to be activated by Dbl (Li and Zheng, 1997). In that context, the development of RhoGEF specific inhibitors would be valuable tools to decipher RhoGTPase signaling pathways. 
As suspected for a long time (Ron et al., 1988), Dbl proteins are involved in various human pathologies including cancer (Kanekura et al., 2004; Orrico et al., 2004). Abnormal exchange factor function was described in various pathologies (Ridley, 2004) in particular due to their oncogenic potential (Karnoub et al., 2004). Therefore, components of RhoGTPase signaling pathways appear as potential anticancer therapeutic targets (Sahai and Marshall, 2002). RhoGTPases have a wide range of tissue distribution, participate in different signaling cascades and can be activated by several exchange factors in the same cell. In contrast, Rho GEFs have a narrower tissue distribution and are activated downstream of specific membrane receptors (Zheng, 2001). Thus, beside their applications for the study of RhoGTPase signaling pathways, RhoGEF inhibitors would also be of major interest to develop new drugs.

Much effort is being made to isolate RhoGEF inhibitors and the interface between the GTPase and the exchange factor may be an interesting target site (Aznar and Lacal, 2001; Ramirez De Molina et al., 2001). Nevertheless, only one RhoGEF specific inhibitor was isolated so far: the peptide TRIP $\alpha$, which inhibits RhoA activation mediated by TRIO-GEFD2, the second exchange domain of TRIO (Schmidt et al., 2002). TRIP $\alpha$ was initially isolated in a two-hybrid screen of a random peptide library according to its ability to bind TRIO-GEFD2 (Schmidt et al., 2002). Structural analyses of the interface between the GTPase and the exchange factor can also help for the design of specific interactors by in silico docking (Cherfils, 2001; Erickson and Cerione, 2004). Applying such structure-based virtual screening approach, a specific inhibitor of Rac activation was identified that targets the RhoGEF recognition groove of the GTPase (Gao et al., 2004). 
We previously reported the Yeast Exchange Assay, a rapid qualitative test to perform wide range screens for GEF specificity (De Toledo et al., 2000). Here we present a novel method based on the Yeast Exchange Assay, which is suitable to perform screens for chemical or peptide inhibitors of GEF activity. To illustrate this application, we present a screen of a library of chemical compounds and the identification of an inhibitor of TRIO-GEFD1, the first exchange domain of TRIO that is an activator of RhoG (Blangy et al., 2000). 


\section{2- Results.}

2.1- RhoG-T17N does not act as a dominant negative to inhibit TRIOGEFD1.

Based on early studies on Ras proteins, the negative T17N mutation is thought to generate a nucleotide-free GTPase that is expected to have a high binding affinity for the exchange factor and thereby to function as a dominant negative mutant (Farnsworth and Feig, 1991; Feig and Cooper, 1988a; Feig and Cooper, 1988b). Accordingly, two hybrid experiments showed that RhoG-T17N bound efficiently to its exchange factor TRIO-GEFD1(Blangy et al., 2000), whereas it did not bind to the RhoA and RhoC exchange factor TRIO-GEFD2 (Figure 1A). When expressed in REF-52 cells, TRIO-GEFD1 induced the formation of lamellipodia and dorsal ruffles (Figure 1B, a-b) characteristic of RhoG activity (Blangy et al., 2000; Vignal et al., 2001). Surprisingly, RhoG-T17N did not inhibit TRIO-GEFD1 induced actin remodeling (Figure 1B, c-e). RhoG-T17N and TRIO-GEFD1 appeared to colocalize in the regions of actin polymerization, in dorsal ruffles and at the leading edge of the cell (arrow in Figure 1B, panels c-e). These results suggest that the two proteins can also interact in fibroblasts but that RhoG-T17N does not act as a competitive dominant negative inhibitor of the exchange factor TRIO-GEFD1. Substitution of D118, another conserved residue among Rho GTPases, also generates a nucleotide binding deficient GTPase as shown for Cdc42 (Tu et al., 2002). According to previous observations (Blangy et al., 2000), RhoG-D118A efficiently inhibited TRIOGEFD1 induced lamellipodia and dorsal ruffles formation (Figure 1B, f-h). Contrarily to RhoG-T17N, RhoG-D118A acted as a dominant negative mutant on TRIO-GEFD1, as shown by the inhibition of actin remodeling. RhoG-T17N thus has an unexpected activity towards TRIO-GEFD1. 
To get a better understanding of these observations in REF-52 cells, we further studied RhoG-T17N and RhoG-D118A in the Yeast Exchange Assay (De Toledo et al., 2000), a GEF assay based on the two hybrid system (Figure 1C, a). Kinectin is a known effector of RhoG (Vignal et al., 2001). When expressed in yeast, RhoG-wt, T17N or -D118A did not bind to kinectin (Figure 1C, b). The further expression of TRIO-GEFD1 promoted the binding of RhoG-wt to kinectin as reported earlier (De Toledo et al., 2000). Unexpectedly, RhoG-T17N also bound to kinectin when TRIOGEFD1 was expressed, whereas RhoG-D118A did not (Figure 1C, b). The binding of RhoG-T17N to kinectin was not observed when TRIO-GEFD2 (Figure 1C, b). Similar results were obtained using two other RhoG effectors unrelated to kinectin (Vignal et al., 2001): the RabGAP RhoGIP56/AS160 (Kane et al., 2002; Larance et al., 2005) and the TRAF3 interacting protein RhoGIP122/T3JAM (Dadgostar et al., 2003; Vigorito et al., 2004), showing that this observation is not restricted to kinectin (our unpublished observations). As TRIO-GEFD1 did not interact directly with kinectin (Figure 1D), these results show that RhoG-T17N can bind to its effectors when the exchange factor TRIO-GEFD1 is expressed.

These observations in yeast are consistent with the results in REF-52 cells and suggest that RhoG-T17N is not an efficient inhibitor of TRIO-GEFD1.

\subsection{Establishment of a GEF inhibitor screening method based on the}

\section{Yeast Exchange Assay.}

To identify RhoGEF inhibitors, we took advantage of the Yeast Exchange Assay. We speculated that in this system we could measure the inhibition of GEF activity by adding a chemical compound or a peptide as a fourth partner (Figure 2A). The only RhoGEF inhibitor described so far is the peptide TRIP $\alpha$ that binds to and 
inhibits TRIO-GEFD2 (Schmidt et al., 2002). In yeast, TRIO-GEFD2 activated RhoC and promoted its binding to its effector ROCK (Figure 2B, a). When TRIP $\alpha$ was expressed as a fourth partner, the binding of RhoC to ROCK was lost (Figure 2B, b). This indicates that TRIPacan inhibit TRIO-GEFD2 activity in yeast. Moreover, TRIP aexpression did not affect RhoC activation by another exchange factor: ARHGEF17 (De Toledo et al., 2000), showing that the peptide is also specific for TRIO-GEFD2 in this assay (Figure 2B). Thus, the Yeast Exchange Assay appears as an appropriate method to screen libraries and identify directly peptides or chemical compounds that interfere with GEF exchange activity.

Yeast has long been regarded as a tempting system to screen for drugs, in particular inhibitors of protein-protein interaction (Vidal and Endoh, 1999). To increase the screening sensitivity for Rho GEF inhibitors, we modified the TAT7 strain to make it permeable to a wider range of chemical compounds including hydrophilic or charged molecules. For that purpose, we inserted a Kanamycin resistance cassette into the erg6 gene. Disruption of erg6, which encodes a methyltransferase in the ergosterol biosynthetic pathway, is not lethal in Saccharomyces cerevisiae. The disruption affects ergosterol synthesis and membrane function, erg6 deficient yeasts being more sensitive to a broad range of compounds among which BrefeldinA, the inhibitor of the ARF GTPase exchange factor Sec7 (Chardin and McCormick, 1999; Shah and Klausner, 1993; Vogel et al., 1993). To verify that erg6 disruption was efficient in the context of the Yeast Exchange Assay, RhoG-wt, Kinectin and TRIO-GEFD1 were expressed in both erg6+ and erg6 yeasts. Cell permeabilization in liquid nitrogen confirmed that $B-$ galactosidase expression induced by TRIO-GEFD1 expression was similar in both yeast strains (Figure $2 \mathrm{C}, \mathrm{a}$ ). We then compared yeast permeability by plating both 
strains on X-gal supplemented medium. Whereas the unmodified TAT7 strain remained white (Figure 2C, b: ERG6+), the strain in which erg6 was disrupted readily turned blue (Figure 2C, b: ERG6-), indicating that the disruption of erg6 helped X-gal entry in yeast.

The disruption of erg6 is compatible with the study of RhoGEF activity in the Yeast Exchange Assay. Moreover, such mutation may facilitate the entry of a wider range of chemical compounds in yeast.

\section{3- Identification of chemical inhibitors of TRIO-GEFD1.}

In order to identify potential TRIO-GEFD1 inhibitors we developed a two-step procedure combining yeast and in vitro exchange assays. We first used the erg $6^{-}$ strain expressing RhoG-wt, Kinectin and TRIO-GEFD1 to screen a series of 3,500 chemicals (see Materials and Methods). Compounds were selected for their ability to inhibit growth in selective $\mathrm{HIS}^{-}$medium, indicative of the inhibition of GTPase activation, and having no effect on growth in non-selective $\mathrm{HIS}^{+}$medium, to discard cytotoxic drugs. Such molecules are candidate inhibitors of TRIO-GEFD1 mediated RhoG activation (Figure 2A). We further tested the selected compounds for their ability to inhibit TRIO-GEFD1 catalyzed nucleotide release by RhoG in vitro, using $\left[{ }^{3} \mathrm{H}\right]-$ GDP dissociation assays with purified recombinant proteins.

Through this procedure, we selected 1-(3-nitrophenyl)-1H-pyrrole-2,5-dione (C10H6N2O4, NPPD, Figure 3A) as a potential TRIO-GEFD1 inhibitor, which was then analyzed in vitro in more details. [ ${ }^{3} \mathrm{H}$-GDP dissociation assays showed that preincubation of TRIO-GEFD1 with NPPD significantly reduced its activity on RhoG, the longer the preincubation, the higher the inhibition (Figure 3B). Preincubation of the inhibitor with the GTPase did not increase its inhibitory activity (our unpublished data). NPPD inhibition was dose dependent. Nucleotide exchange was reduced from 
$70 \%$ down to $55 \%$ in the presence of $50 \mu \mathrm{M}$ NPPD and to only $35 \%$ in the presence of $100 \mu \mathrm{M}$ inhibitor (Figure 3C). Finally, kinetic analysis confirmed that NPPD significantly slowed down the nucleotide exchange reaction catalyzed by TRIOGEFD1 (Figure 3D). These results show that NPPD can function as an inhibitor of TRIO-GEFD1 in vitro.

To identify more potent inhibitors, we tested a series of 23 NPPD structural analogs. We measured the initial exchange rate using $\mathrm{N}$-methylanthraniloyl (Mant)GTP as a fluorescent readout (Hemsath and Ahmadian, 2005). Among the molecules tested two were more efficient inhibitors than NPPD: 1,1'-(1,2-phenylene)bis(1Hpyrrole-2,5-dione) (C14H8N2O4, PEPD, Figure 4A, a) and 1,1'-(2-chloro-1,4phenylene)bis(1H-pyrrole-2,5-dione) (C14H7CIN2O4, CPEPD, Figure 4A, b). At a concentration of $100 \mu \mathrm{M}$, corresponding to a 1000 fold molar excess compared to the exchange factor, NPPD reduced the initial exchange rate by $48.7 \%$, PEPD and CPEPD inhibited the initial exchange rate by 92.4 and $99.8 \%$ respectively (Figure 4B, a-d). Dose response experiments confirmed that PEPD and CPEPD were more efficient than NPPD at inhibiting TRIO-GEFD1 catalyzed nucleotide exchange by RhoG (Figure 4B, a-d): the $\mathrm{IC}_{50}$ of the compounds were $115.5 \mu \mathrm{M}$ for NPPD, $55.8 \mu \mathrm{M}$ for CPEPD and $51 \mu \mathrm{M}$ for PEPD (Figure 4B, d). Of the other analogs of NPPD tested, 10 compounds had a similar inhibitory activity on TRIO-GEFD1 as NPPD and 11 compounds had not effect on the exchange reaction (our unpublished observations).

In the absence of TRIO-GEFD1, Mant-GTP remained stably bound to RhoG in the presence of NPPD, CPEPD or PEPD (Figure 5A, a, b and $c$ respectively) showing that these compounds did not affect the stability of nucleotide binding to RhoG. We finally studied the specificity of the inhibitors. We tested the effects of 
NPPD, PEPD and CPEPD on nucleotide exchange by RhoA catalyzed by ARHGEF17 (Figure 5B, a, b and c respectively) (De Toledo et al., 2000) and by Arf1 catalyzed by ARNO (Figure 5C, a, b and c respectively) (Antonny et al., 1997). In the presence of a 1000 fold molar excess of NPPD (a), CPEPD (b) and PEPD (c) as compared to the exchange factor, the Mant-GTP exchange reactions catalyzed by ARHGEF17 and ARNO were not or slightly inhibited (Figure 5D), as compared to the inhibition of TRIO-GEFD1 by these compounds (Figure 4B, d). 


\section{3- Discussion}

In this report we show that RhoG-T17N does not inhibit its exchange factor TRIO-GEFD1, which illustrates the limitation of using dominant negative RhoGTPases as inhibitors. In order to develop new tools to study RhoGTPase signaling, we developed a novel strategy derived from the Yeast Exchange Assay to screen for inhibitors of exchange factor mediated GTPase activation. Using this approach we further present the screening of a chemical compound library and the identification three chemical inhibitors of TRIO-GEFD1.

Rho GTPases mutants such as Rac1-T17N and Cdc42-T17N have been widely used to inhibit the downstream signaling of exchange factors in vivo. Based on original studies on Ras, these mutants are expected to function by sequestering the exchange factors away from the endogenous GTPase. Several examples do support this model: for instance, expression of Rac1-T17N inhibits membrane ruffling induced by TIAM-1 overexpression (Michiels et al., 1995) and Cdc42-T17N inhibits p38 MAP kinase activation induced by ßPIX overexpression (Lee et al., 2001). Nevertheless, some RhoGTPase T17N mutants may retain the ability to bind their effectors and activate downstream signaling pathways in the presence of an exchange factor, as we illustrate here in the case of RhoG-T17N. This suggests that the stable binding to TRIO-GEFD1 may induce conformational changes allowing RhoG-T17N to bind to its effectors. These observations further support the hypothesis that the binding of the GTPase to the effector may occur within a three partner complex also involving the exchange factor, as suggested for the Dbl, Cdc42 and PAK1 (Wang et al., 2004). This mechanism should participate to the spatial regulation of signaling cascade activation in the cell. We show here that in the presence of TRIO-GEFD1, RhoG$\mathrm{T} 17 \mathrm{~N}$ can bind to its effector, whereas Cdc42-wt but not T17N can form a complex 
with Dbl and PAK1 (Wang et al., 2004). Thus, the T17N mutation may not be equivalent in all RhoGTPases; furthermore, a T17N GTPase may also behave distinctly towards its different GEFs, as reported earlier for other point mutations (Gao et al., 2001; Li et al., 1997; Li and Zheng, 1997). The use of dominant negative mutants also suffers further limitations such as the occurrence of gain of function phenotypes, as shown recently for Cdc42-T17N (Czuchra et al., 2005) and specificity towards the exchange factor. Rho GTPase signaling involves 18 small GTPases and more than 80 RhoGEFs in mammals (Meller et al., 2005; Rossman et al., 2005). Rho GTPases share more than $55 \%$ homology and several RhoGEFs can activate each GTPase. Thus Rho GTPase mutants may be non-discriminative between exchange factors and interfere non-specifically with signaling cascades. In that context, detailed analysis of the complex Rho signaling pathways requires the development of new tools such as specific RhoGEF inhibitors.

We have developed an approach based on the Yeast Exchange Assay, which allows the direct screening of peptide or chemical compounds that inhibit the exchange reaction. We have disrupted the erg6 gene to render yeast sensitive to a wider range of chemical compounds. The disruption is also convenient when performing peptide library screening as it helps rapid identification of interesting peptides: upon plating ERG6 deficient yeasts on X-gal plates, colonies turn blue when the exchange factor is active and stay white when a peptide is expressed that inhibits the exchange reaction. Using this screening procedure, we identified NPPD as a potential inhibitor of TRIO-GEFD1. We confirmed that NPPD inhibits TRIOGEFD1 catalyzed nucleotide exchange by RhoG in vitro. Among chemical analogs of NPPD, we identified CPEPD and PEPD as more efficient inhibitors of TRIO-GEFD1. These compounds did not affect the stability of guanine nucleotide binding on RhoG 
and were able to discriminate between GTPases and exchange factors. Therefore, they appear as good inhibitors of TRIO-GEFD1. We tested a series of 23 analogs of NPPD. The analysis of the active and inactive compounds suggests that the 1(phenyl)-1H-pyrrole-2,5-dione structure is necessary to obtain efficient inhibition of TRIO-GEFD1. Substitutions of small groups at position 4 of the phenyl do not affect inhibition while bigger groups lead to compounds unable to inhibit the exchange reaction. Nevertheless, only 24 compounds were tested and more combinatorial chemistry analyses should be performed to delineate the active backbone and optimize the structures of NPPD, CPEPD and PEPD and further improve their inhibitory effect. Chemical analysis would also be necessary to design molecules with improved solubility and chemical stability.

In conclusion, these results show that the Yeast Exchange Assay is well adapted for high throughput screens of Rho GEF inhibitors and a valuable tool to provide candidate molecules for further studies. Such inhibitors would be most valuable for the study of RhoGTPase signaling pathways and may also have further therapeutic applications. 


\section{4- Materials and methods}

4-1 Plasmids, yeast strain constructs transformation and growth.

The aptamer TRIP $\alpha$ fused to thioredoxin and thioredoxin alone (Schmidt et al., 2002) were fused to the myc tag and inserted with p25MET promoter in pRs422 plasmid that contains the ADE2 selection marker. Arf1 and ARNO proteins were a gift from Bernard Guibert and Jean-Christophe Zeeh, Gif sur Yvette, France. Other yeast, bacteria and cell expression vectors were described earlier (Blangy et al., 2000; De Toledo et al., 2000; Vignal et al., 2001). Yeasts were transformed with LiAc using standard protocols (De Toledo et al., 2000). Yeasts were grown in complete YDP medium or minimal SD medium supplemented with the appropriate aminoacids and containing $0.1 \mathrm{M}$ glucose. For inhibitor screen, yeasts were grown in 96 well plates in selective His- medium containing $2 \mathrm{mM} 3-\mathrm{AT}, 2 \% \mathrm{DMSO}$ and $0.2 \mathrm{mM}$ inhibitor. Each plate was done in duplicate and yeast growth was measured at $\mathrm{OD}_{570 \text {. }}$ All chemical compounds were purchased from ChemBridge (San Diego, CA, USA).

The disruption of erg 6 gene by insertion of a kanamycin resistance cassette was amplified from strain Y00568 (erg6, isogenic to BY4741 with YML008c::kanMX4, a gift from Matthias Peter, Zurich, Switzerland) with the following primers: UPERG6 5'-GCTGTTGCCGATAACTTCTTCATTGC-3' and DWNERG6 5'CtgAtagAAAAtACTgGtCGTTTGCCACG-3', using Platinium Taq Polymerase High Fidelity (Invitrogen). The PCR fragment was then transformed into TAT7 and transformants were selected on plates containing $200 \mu \mathrm{g} / \mathrm{ml} \mathrm{G418.} \mathrm{Disruption} \mathrm{of} \mathrm{erg6}$ gene by homologous recombination was verified by PCR on genomic yeast DNA using primers in erg6 located upstream and downstream UPERG6 and DWNERG6, respectively: UPUPERG6 5'-CGAAGATTGGTGAGAAACCTC-3' and DWNDWNERG6 5'-GTCAATACGTTTGTATGCAGTG-3' as well as primers in the 
kanamycin resistance cassette: KAN787 5'-TTGCCATCCTATGGAACTGC-3' and KAN1023 5'-ACGACTGAATCCGGTGAGAA-3'.

\subsection{Cell lines, transfection and immunofluorescence.}

Rat embryo fibroblasts (REF-52) were cultured at $37^{\circ} \mathrm{C}$ in the presence of $5 \%$ CO2 in DMEM supplemented with $10 \%$ fetal calf serum as described (Blangy et al., 2000). Cells were plated on $12 \mathrm{~mm}$ glass coverslips $16-24$ hours before being transfected using the lipofectamine method as described by the supplier (Invitrogen). 4 hours after transfection, the medium was changed, cells were fixed 20 hours later for 10 minutes in $3.7 \%$ formalin in PBS. After a 2 minute permeabilization in $0.1 \%$ Triton X-100 in PBS and a 30 minute incubation in $0.1 \%$ BSA in PBS, cells were processed for immunofluorescence. Expression of myc-epitope tagged proteins was visualized after incubation with the 9E10 anti-myc monoclonal antibody followed by incubation with biotin conjugated sheep anti mouse IgG (1/200 dilution, Amersham) and then with in streptavidin texas red (1/200 dilution, Amersham). Cells were stained for actin using amino-methyl coumarin (AMCA)-conjugated phalloidin (0.5 $\mathrm{U} / \mathrm{ml}$, Sigma). Cells were washed in PBS and mounted in Mowviol (Aldrich) and observed using a DMR Leica microscope with a 63x planochromat lens. Images were recorded using a Hamamatsu CCD camera, transferred to Adobe Photoshop. Transfections were repeated at least three times and an average of 100 cells were examined.

\subsection{Radioactivity and fluorescence -based guanine nucleotide exchange} assays.

GST-fused GTPases and exchange factors were expressed in E. coli, purified on glutathione-S-Sepharose 4B beads (Amersham) as described previously (Blangy 
et al., 2000; De Toledo et al., 2000). Recombinant Arf1 and ARNO (Antonny et al., 1997) were purified as described (Beraud-Dufour et al., 1998).

Radioactivity based guanine nucleotide exchange assays were performed as described (Blangy et al., 2000; De Toledo et al., 2000). Briefly, $0.3 \mu \mathrm{M}\left[{ }^{3} \mathrm{H}\right] \mathrm{GDP}-$ loaded RhoG was mixed with $1 \mathrm{mM}$ GTP and $0.1 \mu \mathrm{M}$ TRIO-GEFD1 in exchange buffer (50 mM Tris pH7.5, $2 \mathrm{mM} \mathrm{MgCl}$ ) containing 5\% DMSO and the inhibitor at the desired concentration. Reactions were performed at $20^{\circ} \mathrm{C}$. At appropriate time points, reaction was stopped with $50 \mathrm{mM}$ Tris $\mathrm{pH} 7.5,10 \mathrm{mM} \mathrm{MgCl}_{2}$ and the amount of $\left[{ }^{3} \mathrm{H}\right]$ GDP-loaded RhoG was determined by filtration on $0.2 \mu \mathrm{m}$ nitrocellulose as described (Blangy et al., 2000). The GEF activities were presented as radioactivity bound to RhoG relative to samples without GEF defined as $100 \%$.

Fluorescence based guanine nucleotide exchange assays were performed using $\mathrm{N}$ methylanthraniloyl (mant)-GTP (Molecular Probes) in a FLx800 microplate fluorescence reader (BIO-TEK Instruments) at $25^{\circ} \mathrm{C}$. Exchange assays containing $0.1 \mu \mathrm{M}$ GEF were incubated for 30 minutes in reaction buffer $(20 \mathrm{mM}$ Tris- $\mathrm{HCl} \mathrm{pH} 7.5$, $50 \mathrm{mM} \mathrm{NaCl}, 2 \mathrm{mM} \mathrm{MgCl} 2,1 \mu \mathrm{M}$ Mant-GTP and $50 \mu \mathrm{g} / \mathrm{ml} \mathrm{BSA}$ ) containing $5 \%$ DMSO and the inhibitor at the desired concentration. The exchange reaction was started by addition of $1 \mu \mathrm{M}$ GTPase. The relative Mant fluorescence (excitation $=360$ $\mathrm{nm}$, emission $=460 \mathrm{~nm}$ ) was monitored for 10 minutes and measurements were taken every 10 seconds. The rates (kobs) of guanine nucleotide exchange were determined by fitting the data as single exponential association or decay equations using Graphpad Prism 4 as described (Rossman et al., 2003). 


\section{References}

Antonny, B., Beraud-Dufour, S., Chardin, P. and Chabre, M. (1997) N-terminal hydrophobic residues of the G-protein ADP-ribosylation factor-1 insert into membrane phospholipids upon GDP to GTP exchange. Biochemistry (Mosc). 36:4675-84.

Aznar, S. and Lacal, J.C. (2001) Searching new targets for anticancer drug design: the families of Ras and Rho GTPases and their effectors. Prog. Nucleic Acid Res. Mol. Biol. 67:193-234.

Beraud-Dufour, S., Robineau, S., Chardin, P., Paris, S., Chabre, M., Cherfils, J. and Antonny, B. (1998) A glutamic finger in the guanine nucleotide exchange factor ARNO displaces Mg2+ and the beta-phosphate to destabilize GDP on ARF1. EMBO J. 17:3651-9.

Blangy, A., Vignal, E., Schmidt, S., Debant, A., Gauthier-Rouviere, C. and Fort, P. (2000) TrioGEF1 controls Rac- and Cdc42-dependent cell structures through the direct activation of rhoG. J. Cell Sci. 113 ( Pt 4):729-39.

Chardin, P. and McCormick, F. (1999) Brefeldin A: the advantage of being uncompetitive. Cell. 97:153-5.

Cherfils, J. (2001) Structural mimicry of DH domains by Arfaptin suggests a model for the recognition of Rac-GDP by its guanine nucleotide exchange factors. FEBS Lett. 507:280-4.

Czuchra, A., Wu, X., Meyer, H., van Hengel, J., Schroeder, T., Geffers, R., Rottner, K. and Brakebusch, C. (2005) Cdc42 is not essential for filopodium formation, directed migration, cell polarization, and mitosis in fibroblastoid cells. Mol. Biol. Cell. 16:4473-84. 
Dadgostar, H., Doyle, S.E., Shahangian, A., Garcia, D.E. and Cheng, G. (2003) T3JAM, a novel protein that specifically interacts with TRAF3 and promotes the activation of JNK(1). FEBS Lett. 553:403-7.

De Toledo, M., Colombo, K., Nagase, T., Ohara, O., Fort, P. and Blangy, A. (2000) The yeast exchange assay, a new complementary method to screen for Dbllike protein specificity: identification of a novel RhoA exchange factor. FEBS Lett. 480:287-92.

Erickson, J.W. and Cerione, R.A. (2004) Structural elements, mechanism, and evolutionary convergence of Rho protein-guanine nucleotide exchange factor complexes. Biochemistry (Mosc). 43:837-42.

Etienne-Manneville, S. and Hall, A. (2002) Rho GTPases in cell biology. Nature. 420:629-35.

Farnsworth, C.L. and Feig, L.A. (1991) Dominant inhibitory mutations in the $\mathrm{Mg}(2+)-$ binding site of RasH prevent its activation by GTP. Mol. Cell. Biol. 11:4822-9.

Feig, L.A. and Cooper, G.M. (1988a) Inhibition of NIH 3T3 cell proliferation by a mutant ras protein with preferential affinity for GDP. Mol. Cell. Biol. 8:3235-43.

Feig, L.A. and Cooper, G.M. (1988b) Relationship among guanine nucleotide exchange, GTP hydrolysis, and transforming potential of mutated ras proteins. Mol. Cell. Biol. 8:2472-8.

Gao, Y., Dickerson, J.B., Guo, F., Zheng, J. and Zheng, Y. (2004) Rational design and characterization of a Rac GTPase-specific small molecule inhibitor. Proc. Natl. Acad. Sci. U. S. A. 101:7618-23.

Gao, Y., Xing, J., Streuli, M., Leto, T.L. and Zheng, Y. (2001) Trp(56) of rac1 specifies interaction with a subset of guanine nucleotide exchange factors. J. Biol. Chem. 276:47530-41. 
Hemsath, L. and Ahmadian, M.R. (2005) Fluorescence approaches for monitoring interactions of Rho GTPases with nucleotides, regulators, and effectors. Methods. 37:173-82.

Kane, S., Sano, H., Liu, S.C., Asara, J.M., Lane, W.S., Garner, C.C. and Lienhard, G.E. (2002) A method to identify serine kinase substrates. Akt phosphorylates a novel adipocyte protein with a Rab GTPase-activating protein (GAP) domain. J. Biol. Chem. 277:22115-8.

Kanekura, K., Hashimoto, Y., Niikura, T., Aiso, S., Matsuoka, M. and Nishimoto, I. (2004) Alsin, the product of ALS2 gene, suppresses SOD1 mutant neurotoxicity through RhoGEF domain by interacting with SOD1 mutants. J. Biol. Chem. 279:19247-56.

Karnoub, A.E., Symons, M., Campbell, S.L. and Der, C.J. (2004) Molecular basis for Rho GTPase signaling specificity. Breast Cancer Res. Treat. 84:61-71.

Larance, M., Ramm, G., Stockli, J., van Dam, E.M., Winata, S., Wasinger, V., Simpson, F., Graham, M., Junutula, J.R., Guilhaus, M. and James, D.E. (2005) Characterization of the role of the Rab GTPase-activating protein AS160 in insulin-regulated GLUT4 trafficking. J. Biol. Chem. 280:37803-13.

Lee, S.H., Eom, M., Lee, S.J., Kim, S., Park, H.J. and Park, D. (2001) BetaPixenhanced p38 activation by Cdc42/Rac/PAK/MKK3/6-mediated pathway. Implication in the regulation of membrane ruffling. J. Biol. Chem. 276:2506672.

Li, R., Zhang, B. and Zheng, Y. (1997) Structural determinants required for the interaction between Rho GTPase and the GTPase-activating domain of p190. J. Biol. Chem. 272:32830-5. 
Li, R. and Zheng, Y. (1997) Residues of the Rho family GTPases Rho and Cdc42 that specify sensitivity to Dbl-like guanine nucleotide exchange factors. J. Biol. Chem. 272:4671-9.

Meller, N., Merlot, S. and Guda, C. (2005) CZH proteins: a new family of Rho-GEFs. J. Cell Sci. 118:4937-46.

Michiels, F., Habets, G.G., Stam, J.C., van der Kammen, R.A. and Collard, J.G. (1995) A role for Rac in Tiam1-induced membrane ruffling and invasion. Nature. 375:338-40.

Orrico, A., Galli, L., Cavaliere, M.L., Garavelli, L., Fryns, J.P., Crushell, E., Rinaldi, M.M., Medeira, A. and Sorrentino, V. (2004) Phenotypic and molecular characterisation of the Aarskog-Scott syndrome: a survey of the clinical variability in light of FGD1 mutation analysis in 46 patients. Eur. J. Hum. Genet. 12:16-23.

Ramirez De Molina, A., Rodriguez-Gonzalez, A. and Lacal, J.C. (2001) Targeting new anticancer drugs within signalling pathways regulated by the Ras GTPase superfamily (Review). Int. J. Oncol. 19:5-17.

Ridley, A.J. (2004) Rho proteins and cancer. Breast Cancer Res. Treat. 84:13-9.

Ron, D., Tronick, S.R., Aaronson, S.A. and Eva, A. (1988) Molecular cloning and characterization of the human dbl proto-oncogene: evidence that its overexpression is sufficient to transform NIH/3T3 cells. EMBO J. 7:2465-73.

Rossman, K.L., Cheng, L., Mahon, G.M., Rojas, R.J., Snyder, J.T., Whitehead, I.P. and Sondek, J. (2003) Multifunctional roles for the PH domain of Dbs in regulating Rho GTPase activation. J. Biol. Chem. 278:18393-400. 
Rossman, K.L., Der, C.J. and Sondek, J. (2005) GEF means go: turning on RHO GTPases with guanine nucleotide-exchange factors. Nat Rev Mol Cell Biol. 6:167-80.

Sahai, E. and Marshall, C.J. (2002) RHO-GTPases and cancer. Nat Rev Cancer. 2:133-42.

Schmidt, S., Diriong, S., Mery, J., Fabbrizio, E. and Debant, A. (2002) Identification of the first Rho-GEF inhibitor, TRIPalpha, which targets the RhoA-specific GEF domain of Trio. FEBS Lett. 523:35-42.

Shah, N. and Klausner, R.D. (1993) Brefeldin A reversibly inhibits secretion in Saccharomyces cerevisiae. J. Biol. Chem. 268:5345-8.

Tu, S.S., Wu, W.J., Yang, W., Nolbant, P., Hahn, K. and Cerione, R.A. (2002) Antiapoptotic Cdc42 mutants are potent activators of cellular transformation. Biochemistry. 41:12350-8.

Vidal, M. and Endoh, H. (1999) Prospects for drug screening using the reverse twohybrid system. Trends Biotechnol. 17:374-81.

Vignal, E., Blangy, A., Martin, M., Gauthier-Rouviere, C. and Fort, P. (2001) Kinectin is a key effector of RhoG microtubule-dependent cellular activity. Mol. Cell. Biol. 21:8022-34.

Vigorito, E., Bell, S., Hebeis, B.J., Reynolds, H., McAdam, S., Emson, P.C., McKenzie, A. and Turner, M. (2004) Immunological function in mice lacking the Rac-related GTPase RhoG. Mol. Cell. Biol. 24:719-29.

Vogel, J.P., Lee, J.N., Kirsch, D.R., Rose, M.D. and Sztul, E.S. (1993) Brefeldin A causes a defect in secretion in Saccharomyces cerevisiae. J. Biol. Chem. 268:3040-3. 
Wang, L., Zhu, K. and Zheng, Y. (2004) Oncogenic Dbl, Cdc42, and p21-activated kinase form a ternary signaling intermediate through the minimum interactive domains. Biochemistry (Mosc). 43:14584-93.

Zheng, Y. (2001) Dbl family guanine nucleotide exchange factors. Trends Biochem. Sci. 26:724-32. 


\section{Figure legends}

Figure 1: RhoG-T17N mutant does not inhibit the exchange factor TRIOGEFD1. A: Two-hybrid protein interactions. Interaction between RhoG-wt, -G12V or T17N fused to LexA DNA binding domain (LexA) and TRIO-GEFD1 (GEFD1, aa 1232-1429), TRIO-GEFD2 (GEFD2, aa 1849-2450) or Kinectin (aa 630-935) fused to GAL4 activation domain (GAL-AD) was visualized in TAT7 by monitoring the lacZ reporter gene expression using a filter assay for ß-galactosidase activity. B: Effects of RhoG-T17N and -D118A on TRIO-GEFD1 induced actin reorganization. REF-52 cells expressing myc tagged TRIO-GEFD1 alone ( $a$ and $b$ ) or with GFP tagged RhoG-T17N (c-e) or -D118A (f-h) were processed for detection of myc epitope (a and d), GFP fluorescence (c) and filamentous actin (b and e). Bar in b, $10 \mu \mathrm{M}$. C: Yeast Exchange Assay. (a) Principle of the yeast exchange assay (De Toledo et al., 2000). Wild type GTPase does not bind to its effector and the ß-galactosidase reporter gene is not expressed unless a specific GEF is expressed (b) B-galactosidase activity in TAT7 expressing RhoG-wt, -T17N, -D118A or -G12V fused to LexA DNA binding domain, Kinectin RhoG binding domain fused to GAL4 activation domain and no exchange factor (none) or myc tagged TRIO-GEFD1 (GEFD1) or TRIO-GEFD2 (GEFD2). D: Two-hybrid protein interactions performed as in A. Interaction between RhoG-T17N and kinectin fused to LexA DNA binding domain (LexA) and TRIOGEFD1 or Kinectin (aa 630-935) fused to GAL4 activation domain (GAL-AD)

Figure 2: Adaptations of the Yeast Exchange Assay to isolate Rho GEF inhibitors. A: Principle of the GEF inhibitor detection system based on the Yeast Exchange Assay. Wild type GTPase does not bind to its effector and reporter genes are not expressed (a) unless a specific GEF is expressed (b). Addition of a peptide or a chemical compound that inhibit the GEF results in GTPase inactivation and the lack 
of expression of the reporter genes (c). B: Specific inhibition of TRIO-GEFD2 by the TRIPapeptide in the Yeast Exchange Assay. ß-galactosidase activity in TAT7 expressing RhoC-wt fused to LexA DNA binding domain, GAL4 activation domain fused to ROCK, no GEF (none) or myc tagged TRIO-GEFD2 or ARHGEF17 and myc tagged thioredoxin $(a:-\operatorname{TRIP} \alpha)$ or thioredoxin fused to TRIP $\alpha(b:+\operatorname{TRIP} \alpha)$. C: The disruption of erg6 allows X-gal entry into yeast. Detection of ß-galactosidase activity with a filter assay after yeast breakage with liquid nitrogen (a) or directly on X-gal containing plates (b) in TAT7 yeast bearing the erg6 gene disruption (ERG6-) or not (ERG6+) and expressing RhoG-wt fused to LexA DNA binding domain, GAL4 activation domain fused Kinectin and no GEF (none) or myc tagged TRIO-GEFD1.

Figure 3: Characterization of 1-(3-nitrophenyl)-1H-pyrrole-2,5-dione as a TRIO-GEFD1 inhibitor. In vitro exchange assays were performed by measuring the decrease with time of radioactivity associated with RhoG. The data shown are representative of three individual experiments. Means and SEM are shown for duplicate measures. A: Structure of 1-(3-nitrophenyl)-1H-pyrrole-2,5-dione (NPPD), B: $0.1 \mu \mathrm{M}$ TRIO-GEFD1 (GEFD1) was incubated for 30 minutes in exchange buffer with $5 \%$ DMSO or for 30,15 or 5 minutes in 5\% DMSO containing $100 \mu$ M NPPD. 0.3 $\mu \mathrm{M}\left[{ }^{3} \mathrm{H}\right]$-GDP loaded RhoG and $1 \mathrm{mM}$ GTP were then added and the amount of radioactivity associated with the GTPase was measured after 15 minutes. $100 \%$ was determined by incubating $\left[{ }^{3} \mathrm{H}\right]-$ GDP loaded RhoG alone in exchange buffer containing $1 \mathrm{mM}$ GTP and 5\% DMSO for 15 minutes (-GEF). C: $0.1 \mu \mathrm{M}$ TRIOGEFD1 (GEFD1) was incubated for 30 minutes in exchange buffer containing $5 \%$ DMSO alone or with $50 \mu \mathrm{M}$ or $100 \mu \mathrm{M}$ NPPD. $0.3 \mu \mathrm{M}\left[{ }^{3} \mathrm{H}\right]$-GDP loaded RhoG and 1 mM GTP were then added and the amount of radioactivity associated with RhoG was measured after 5 minutes. $100 \%$ was determined by incubating $\left[{ }^{3} \mathrm{H}\right.$ ]-GDP loaded 
RhoG alone in exchange buffer containing $1 \mathrm{mM}$ GTP and $5 \%$ DMSO for 5 minutes (GEF). D: $0.1 \mu \mathrm{M}$ TRIO-GEFD1 was incubated for 30 minutes in exchange buffer with $5 \%$ DMSO (squares) or 5\% DMSO containing $100 \mu \mathrm{M}$ NPPD (lozenges). $0.3 \mu \mathrm{M}\left[{ }^{3} \mathrm{H}\right]-$ GDP loaded RhoG and $1 \mathrm{mM}$ GTP were then added and the amount of radioactivity associated with RhoG was measured after $0,1,2,5,10$ and 15 minutes. As a control, [ $\left.{ }^{3} \mathrm{H}\right]$-GDP loaded RhoG was incubated alone in exchange buffer containing 1 mM GTP and 5\% DMSO (triangles).

Figure 4: Analysis of TRIO-GEFD1 inhibition by NPPD, PEPD and CPEPD. In vitro exchange assays were performed by measuring the increase in fluorescence emitted with time upon incorporation of Mant-GTP into RhoG. A: Structure of 1,1'(1,2-phenylene)bis(1H-pyrrole-2,5-dione) (PEPD, a) and 1,1'-(2-chloro-1,4phenylene)bis(1H-pyrrole-2,5-dione) (CPEPD, b). B: $0.1 \mu \mathrm{M}$ TRIO-GEFD1 was incubated with $1 \mu \mathrm{M}$ RhoG in the presence of NPPD (a), CPEPD (b) and PEPD (c) at $0 \mu \mathrm{M}$ (DMSO), $25 \mu \mathrm{M}, 50 \mu \mathrm{M}$ and $100 \mu \mathrm{M}$. (d) $\mathrm{K}_{\mathrm{obs}}$, inhibition of the exchange reactions shown in a-c and $\mathrm{IC}_{50}$ of the compounds.

Figure 5: Analysis of NPPD, PEPD and CPEPD specificity. In vitro exchange assays were performed by measuring the amount in fluorescence emitted with time upon binding of Mant-GTP into RhoG. A: $1 \mu \mathrm{M}$ of Mant-GTP loaded RhoG was incubated alone (DMSO in a-c) or with $100 \mu \mathrm{M}$ NPPD (a), CPEPD (b) or PEPD (c) or with the exchange factor (TRIO-GEFD1 in a-C). B: $1 \mu \mathrm{M}$ RhoA was incubated alone (no GEF, a-c) or with $0.1 \mu \mathrm{M}$ ARHGEF17 alone (DMSO in a-c) or in the presence NPPD (a), CPEPD (b) and PEPD (b) at $100 \mu \mathrm{M}$, corresponding to a 1000 fold molar excess compared to the exchange factor. C: $1 \mu \mathrm{M}$ Arf1 was incubated alone (no GEF, a-c) or with $0.02 \mu \mathrm{M}$ ARNO alone (DMSO in a-c) or in the presence NPPD (a), CPEPD (b) and PEPD (b) at $20 \mu \mathrm{M}$, corresponding to a 1000 fold molar excess 
compared to the exchange factor. $\mathbf{D}: \mathrm{K}_{\mathrm{obs}}$ and inhibition of the exchange reactions shown in B (RhoA+ARHGEF17) and C (Arf1+ARNO). 


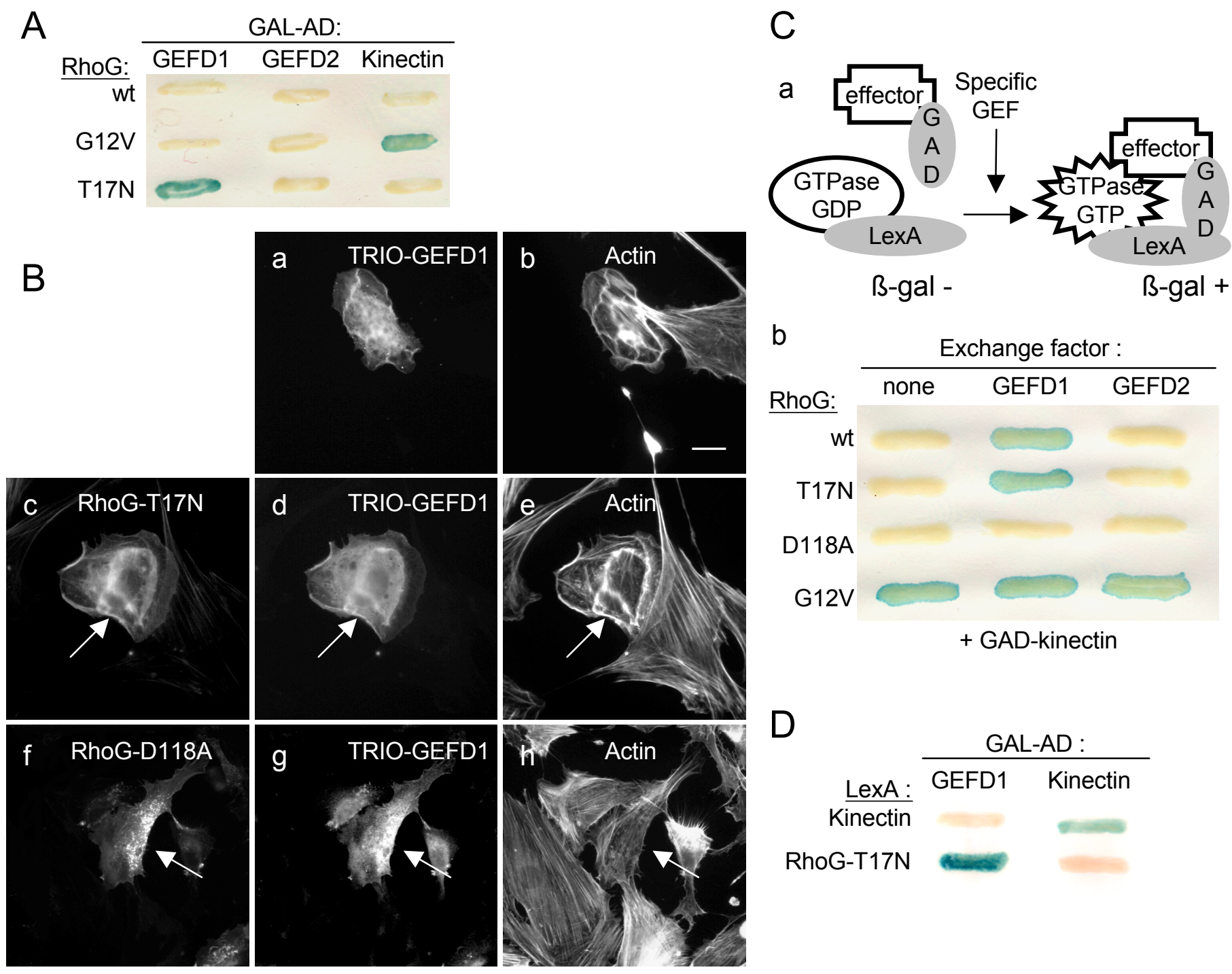


A a

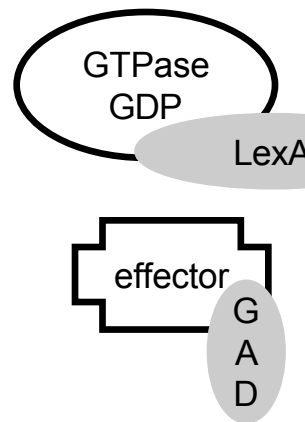

ß-gal -

HIS- b

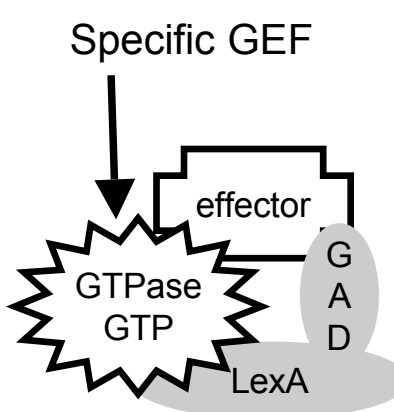

ß-gal +

HIS+

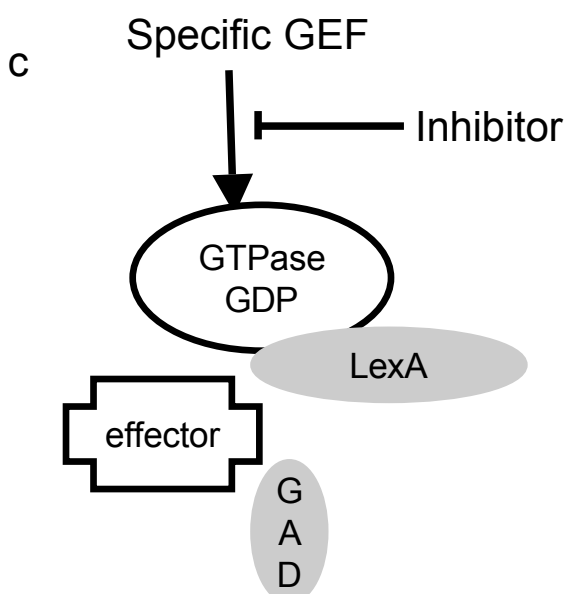

ß-gal HIS-

B

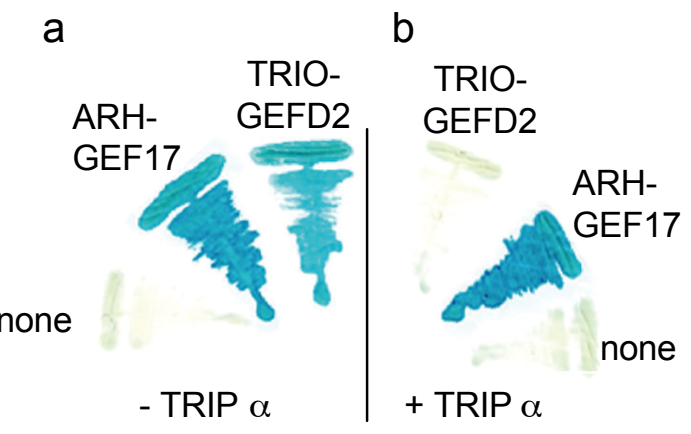

C

a

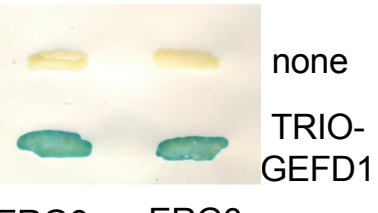

ERG6+ ERG6- 
A

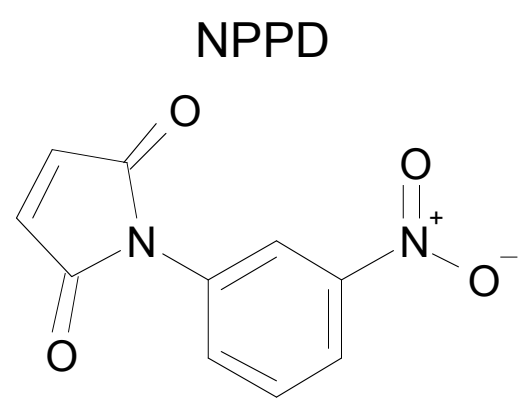

1-(3-nitrophenyl)-1H-pyrrole-2,5-dione

C

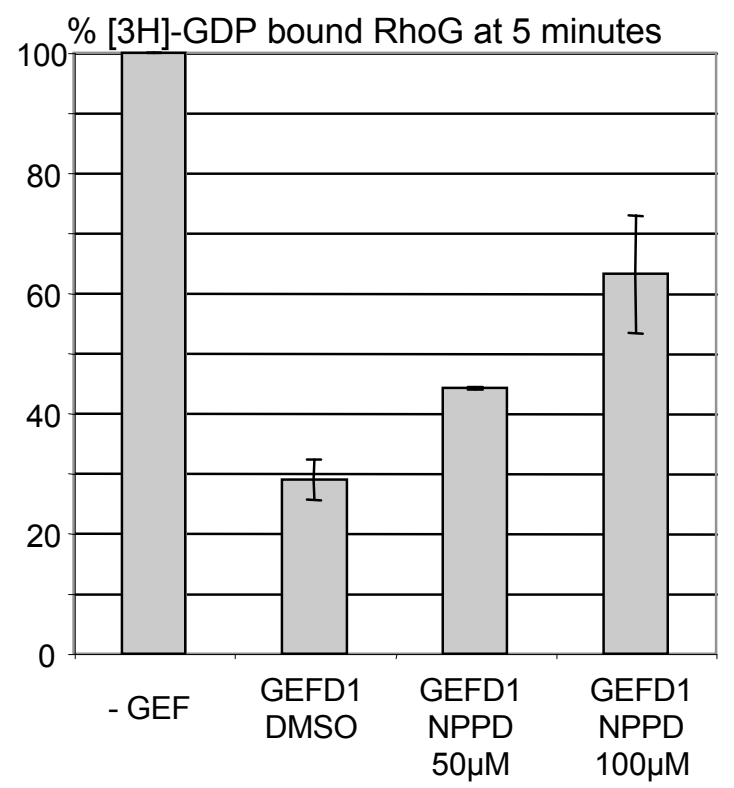

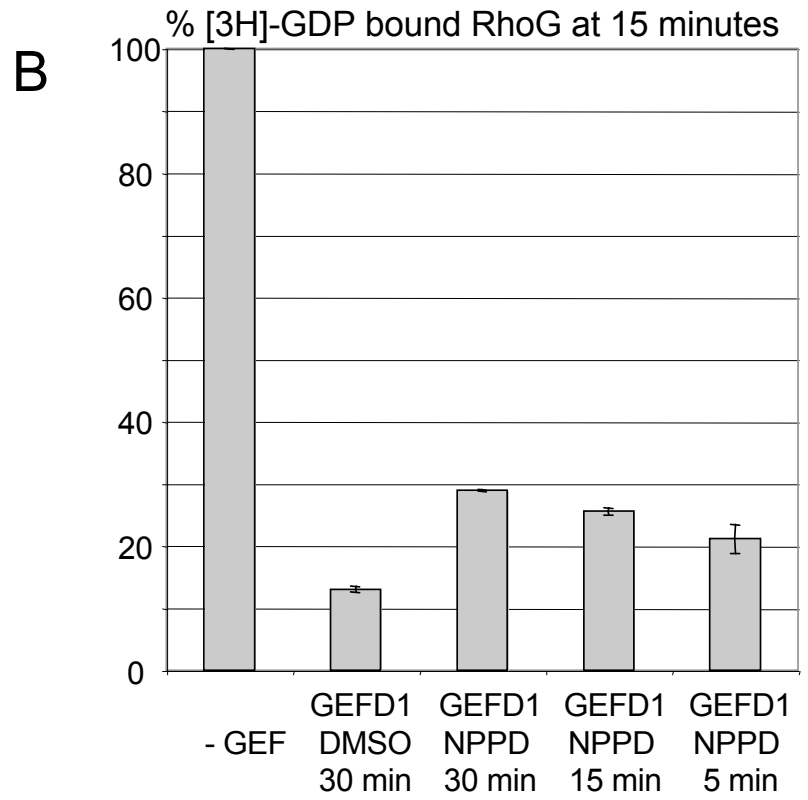

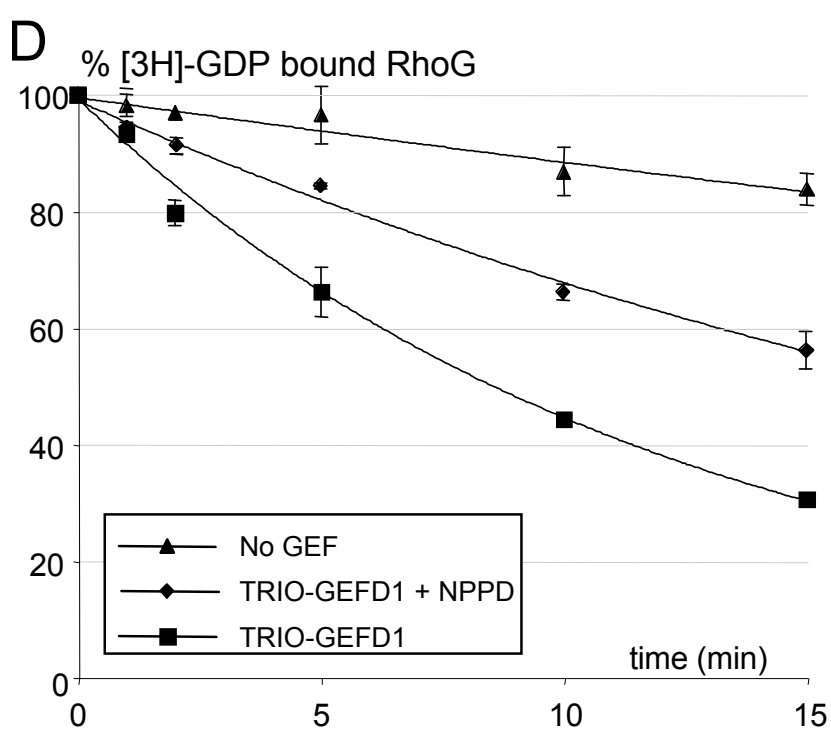




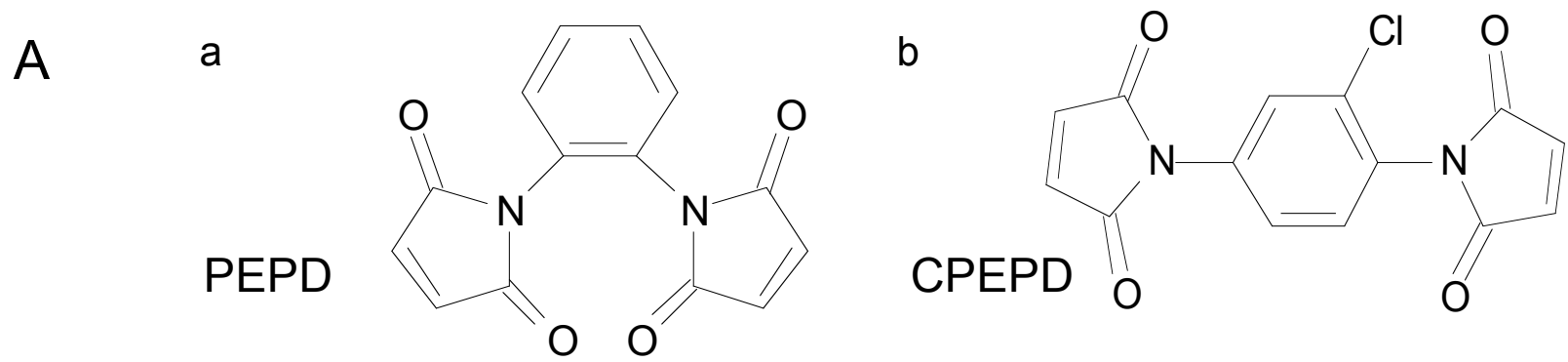

1,1'-(1,2-phenylene)bis(1H-pyrrole-2,5-dione) 1,1'-(2-chloro-1,4-phenylene)bis(1H-pyrrole-2,5-dione)
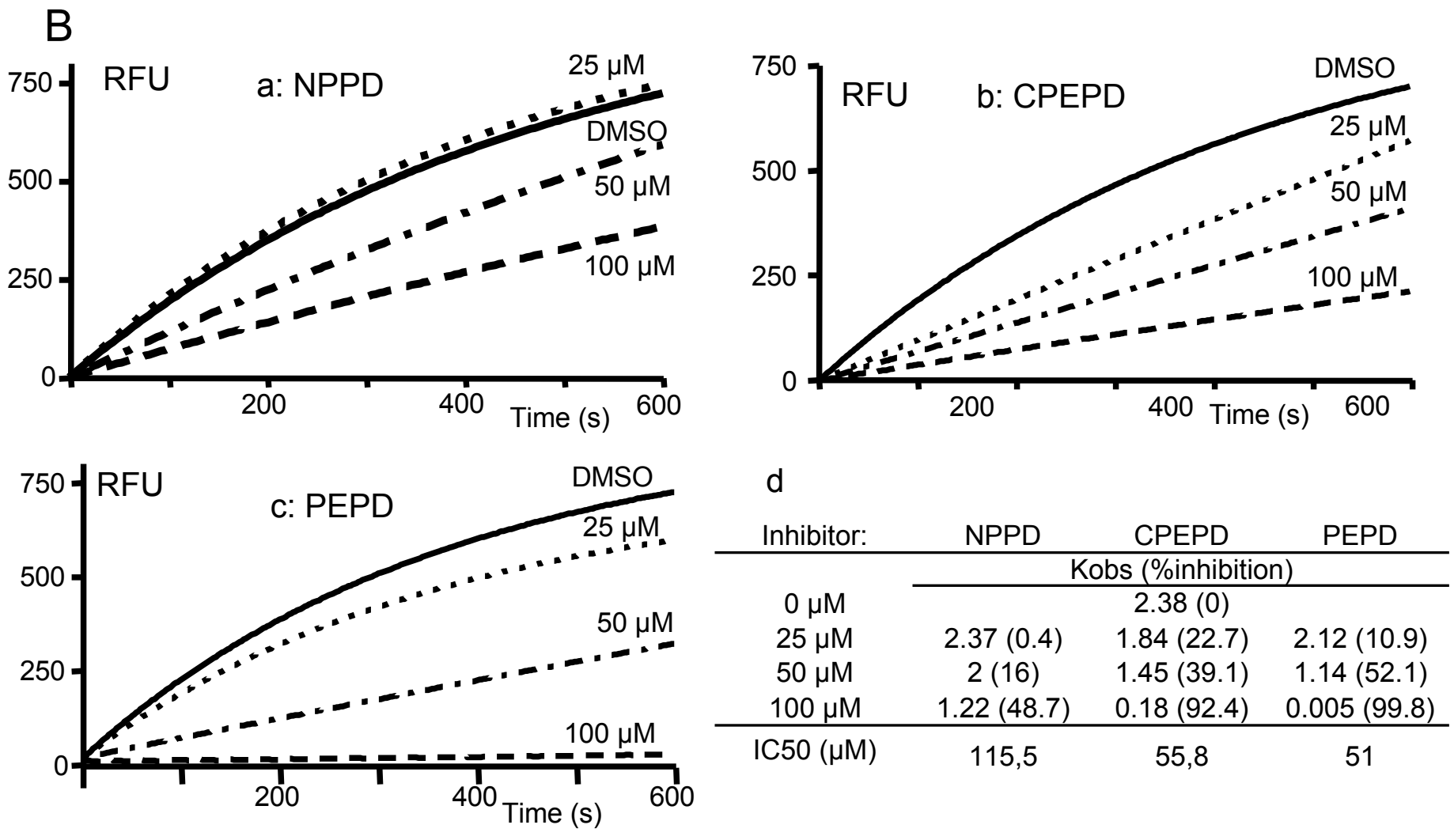
A a: NPPD

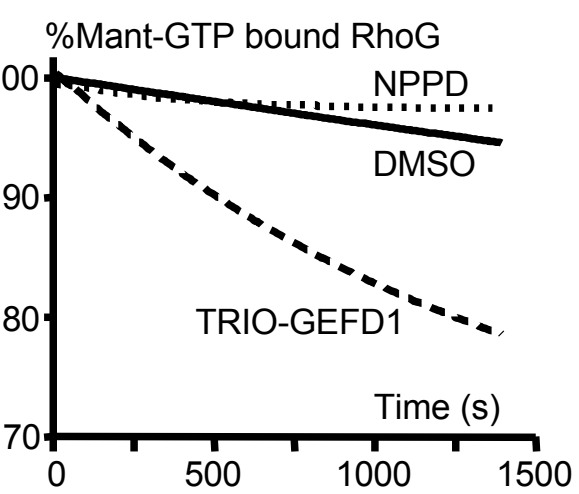

b: CPEPD

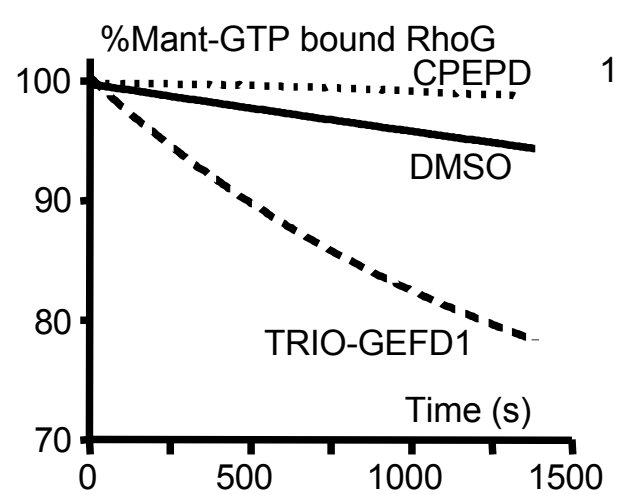

c: PEPD

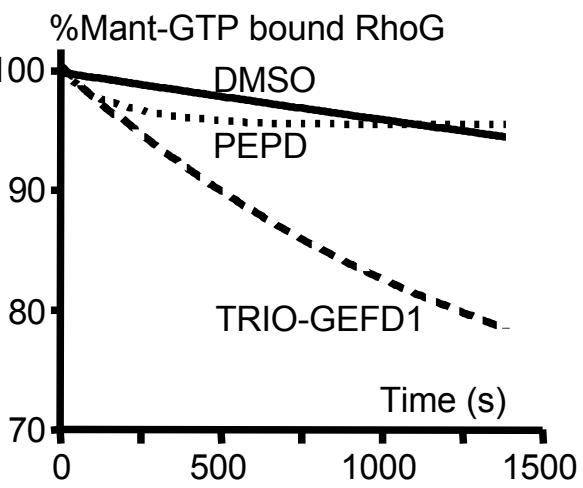

\section{B RhoA +ARHGEF17}
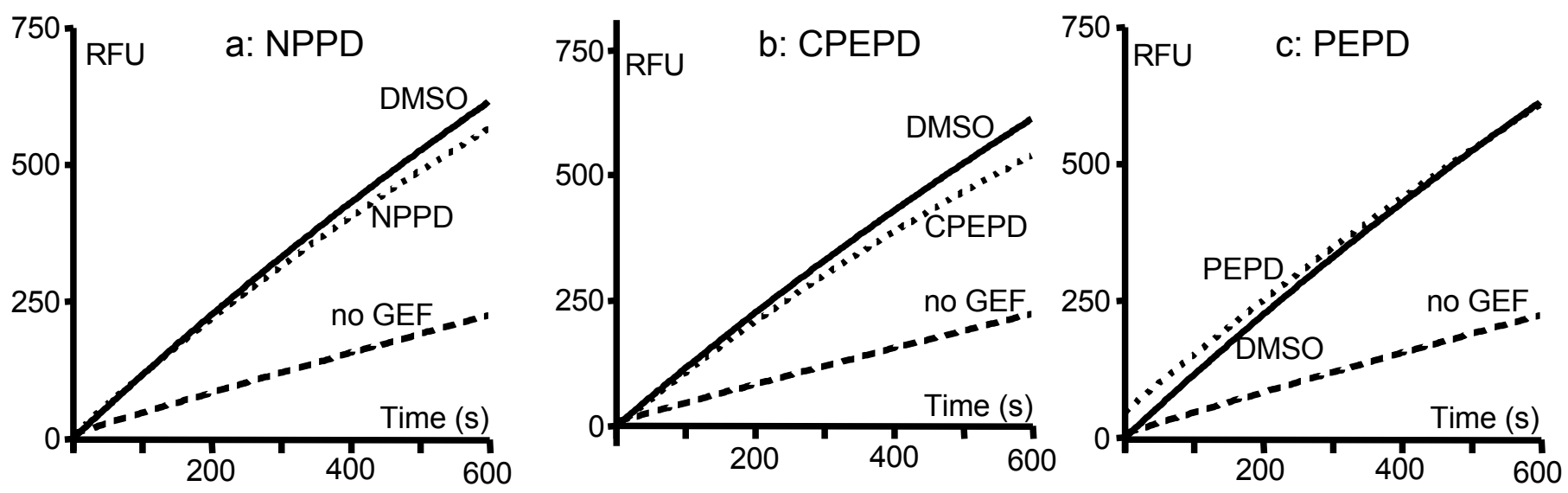

\section{Arf1 + ARNO}

a: NPPD

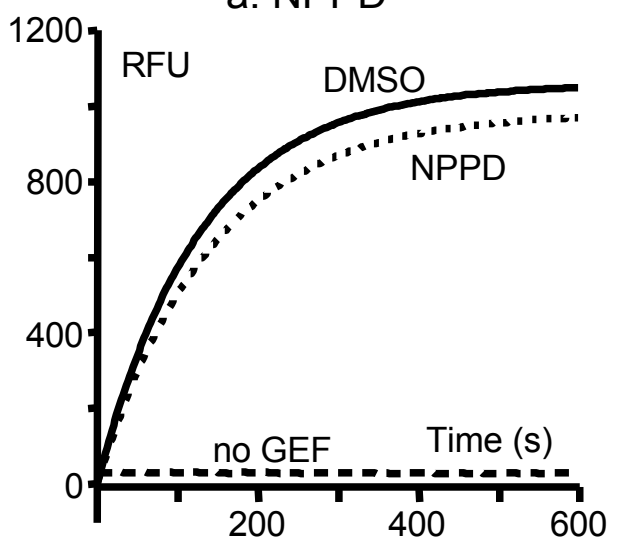

b: CPEPD

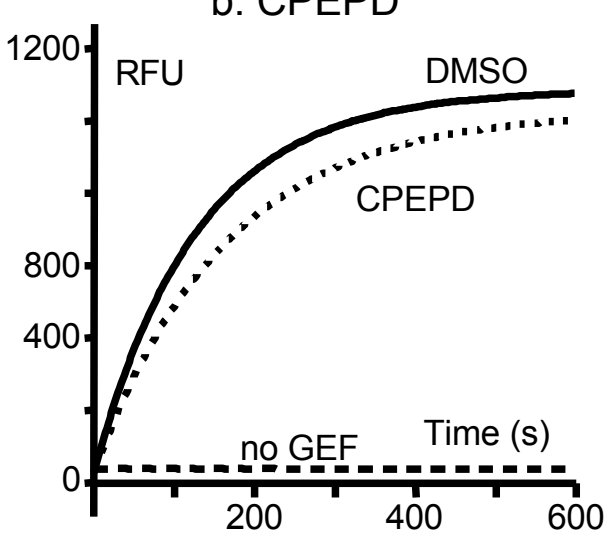

c: PEPD

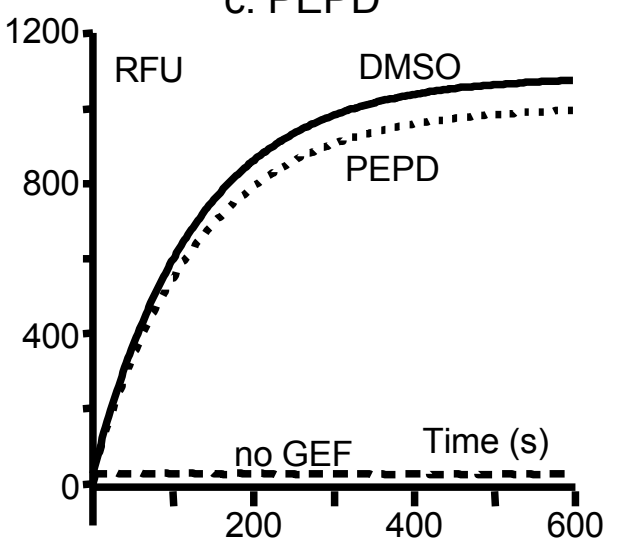

$\mathrm{K}_{\text {obs }}$ (\%inhibition)

\begin{tabular}{ccccc} 
Inhibitor: & none & NPPD & CPEPD & PEPD \\
\cline { 2 - 5 } & & & & \\
GTPase+GEF: & & & \\
RhoA+ARHGEF17 & $1.0(0)$ & $0.93(9.2)$ & $0.94(8.3)$ & $0.90(11.7)$ \\
Arf1+ARNO & $7.76(0)$ & $7.16(7.7)$ & $6.14(20.9)$ & $7.6(2.1)$
\end{tabular}

